University of Tennessee Health Science Center UTHSC Digital Commons

Spring 4-13-2021

\title{
The Use of DMAIC to Improve Quality Vaccination Recommendations in Chain Community Pharmacies
}

\author{
Urvi Patel \\ University of Tennessee Health Science Center
}

Follow this and additional works at: https://dc.uthsc.edu/hiimappliedresearch

Part of the Health and Medical Administration Commons, and the Health Information Technology Commons

\section{Recommended Citation}

Patel, Urvi, "The Use of DMAIC to Improve Quality Vaccination Recommendations in Chain Community Pharmacies" (2021). Applied Research Projects. 74. . https://doi.org/10.21007/chp.hiim.0071

https://dc.uthsc.edu/hiimappliedresearch/74

This Research Project is brought to you for free and open access by the Department of Health Informatics and Information Management at UTHSC Digital Commons. It has been accepted for inclusion in Applied Research Projects by an authorized administrator of UTHSC Digital Commons. For more information, please contact jwelch30@uthsc.edu. 
The Use of DMAIC to Improve Quality Vaccination Recommendations in Chain Community

Pharmacies

The Use of DMAIC to Improve Quality Vaccination Recommendations in Chain Community Pharmacies

\author{
Urvi Patel, BSPS, PharmD Candidate \\ University of Tennessee Health Science Center \\ Master of Health Informatics and Information Management \\ Advisor: Sajeesh Kumar, $\mathrm{PhD}$
}

April 2021

IRB 18-06415-XP 
Table of Contents

Acknowledgments...........................................

Abstract...................................................4

Keywords............................................4

Abbreviations..............................................4

\section{Chapter 1:}

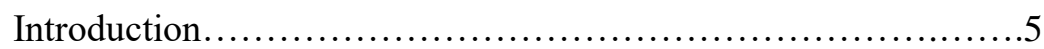

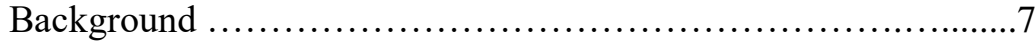

Chapter 2:

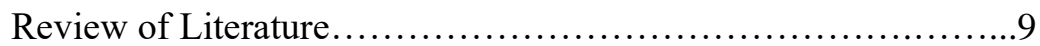

\section{Chapter 3:}

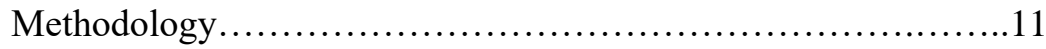

Research Question............................................11

Population \& Study Methods.................................11

\section{Chapter 4:}

LSS and The DMAIC Methodology ............................12

\section{Chapter 5:}

DMAIC Case Study ........................................ 14

Define .................................................. 14

Measure..................................................14

Analysis..................................................... 15

Implement...............................................16

Results of Implementation...................................17

Control...................................................20

\section{Chapter 6:}

Discussion.................................................21

Limitations and Insights.................................21

Conclusion.................................................23

References.................................................... 25 
The Use of DMAIC to Improve Quality Vaccination Recommendations in Chain Community

\section{Acknowledgments}

The completion of this project could not have been possible without the help and efforts of my research team at the University of Tennessee Health Science Center College of Pharmacy. They have collectively helped create this research case study. First, I would like to thank the lead researchers of the project: Justin Gatwood, Ph.D. and Kenneth Hohmeier, PharmD, for their mentorship with the IRB process and their work on this project to investigate vaccine hesitancy and implement an assertive training program to improve pneumococcal vaccinations in the state of Tennessee. I would like to express my sincere gratitude to Dean Marie Chisholm-Burns, PharmD, MPH, MBA, FCCP, FASHP, FAST of the College of Pharmacy for her inspiration, guidance, and the opportunity to engage in a research project focused on Lean Six Sigma. Furthermore, I would like to thank Alina Cernasev, Ph.D., PharmD, for helping me gather qualitative data and conduct interviews. I would also like to thank my peers, Connor Alexander, B.S, and Matthew Cheramie, B.S, for their contribution in analyzing data and constructing the statistical figures included in this paper. Lastly and humbly, I would like to thank Sajeesh Kumar, Ph.D., for his mentorship and guidance on the thesis, which has allowed me to present the completed work to the College of Health Professions at the University of Tennessee Health Science Center. 
The Use of DMAIC to Improve Quality Vaccination Recommendations in Chain Community Pharmacies

\begin{abstract}
Community pharmacies provide the convenience and ease of administrating vaccinations outside traditional settings. Vaccinations are health initiatives that protect communities and improve health outcomes in all populations. Despite their accessibility and supporting clinical data, various influential factors contribute to the current suboptimal rates of vaccine administration. Given the common barriers to vaccine administration, this research narrows down to address a specific barrier and attempts to implement a method that focuses on improving vaccine rates in community pharmacies. This research is a case study that utilizes the DMAIC model of lean six sigma and aims to use this quality improvement process to identify, measure, analyze, and implement a training program to facilitate pharmacists in high-quality vaccine recommendations to promote higher rates of pneumococcal vaccinations in community settings.
\end{abstract}

Keywords: pneumococcal vaccine, vaccine hesitancy, lean six sigma, DMAIC, pharmacists, Walgreens, quality improvement.

Abbreviations:

LSS: Lean Six Sigma

DMAIC: Define, Measure, Analyze, Implement, Control

PCV13: pneumococcal conjugate vaccine

PPSV23: pneumococcal polysaccharide vaccine 
The Use of DMAIC to Improve Quality Vaccination Recommendations in Chain Community Pharmacies

The Use of DMAIC to Improve Quality Vaccination Recommendations in Chain Community Pharmacies

\section{Chapter 1: Introduction}

Vaccinations are vital preventative tools to help protect and significantly reduce the risk of various medical illnesses and disease states in the community. Pharmacists play a crucial role in impacting vaccination rates as certified immunizers and patient health advocates. Seen in multiple settings, $50 \%-90 \%$ of patients follow a pharmacist's advice and accept the recommended vaccine (Grabenstein, 1998). A 2011 study conducted by Taitel et al. showed that $4.88 \%$ of the 1.3 million high-risk patients immunized by a pharmacist received a pneumococcal vaccine; this value is significantly greater than the national mark $2.90 \%$ (2011). However, one significant barrier to utilizing a new pharmacy service is the lack of time for pharmacists to implement and monitor the service (Powers \& Bright, 2008). One way to overcome this barrier is to utilize technicians for tasks that do not require a pharmacist's professional training. Another barrier specific to a new vaccination service within a pharmacy is the design of reimbursement. Many patients do not have health insurance plans that cover the cost of vaccinations administered outside of a primary care provider's office (“Addressing barriers,” 2018). This may limit the number of patients eligible to receive a vaccination within the pharmacy. Health practitioners have tried to understand the basis of vaccination hesitancy and found several strategies have been implemented to reduce hesitancy, such as improving the quality of vaccine education to patients, clearing misconceptions, decreasing wait times, improving accessibility, and making strong recommendations. In many community pharmacy settings, while pharmacists are the first-line practitioners to advocate for providing scheduled vaccinations, a deeper layer of 
The Use of DMAIC to Improve Quality Vaccination Recommendations in Chain Community

hesitancy has been observed. In many instances, within such patient-driven yet dynamic environments, passive pharmacist engagement and the lack of active attention in recommending vaccinations to patients have been observed. They have contributed to current suboptimal pneumococcal vaccination rates.

Despite having clinically available and effective vaccinations to protect and prevent the public and susceptible patient populations, barriers exist that prevent immunization rate goal achievement of $90 \%$ established by Healthy People 2020. These barriers include but are not limited to vaccine hesitancy, lack of access, high costs, vaccine misconceptions, lack of education, fear of needles, and related factors (MacDonald et al., 2018). Many approaches have been proposed to overcome these barriers, including providing patient educational material, using proven communication strategies, understanding and clearing misconceptions, improving accessibility for vaccination, increasing provider engagement, and making strong recommendations.

This study aims to report how a lean six sigma (LSS) DMAIC approach can be used to address specific barriers of hesitancy and vaccination rates, mainly focusing on pneumococcal vaccination rates. An assertive training program to educate and train community pharmacists on vaccine assertiveness techniques and skills focusing on increasing pneumococcal vaccination recommendations in adult patient populations will be described. The primary outcome is the positive change in pneumococcal vaccination rates before and after the intervention. 
The Use of DMAIC to Improve Quality Vaccination Recommendations in Chain Community

\section{Background}

Pneumococcal disease, an infectious disease caused by the bacteria Streptococcus pneumonia (S. pneumoniae), is responsible for high incidences of morbidity and mortality each year in the United States and worldwide. Pneumococcal disease has the ability to cause sinusitis, otitis media, pneumonia, meningitis, and sepsis leading to severe and detrimental outcomes. The clinical presentation of pneumococcal disease typically results from bacterial colonization of the upper respiratory tract primarily via respiratory droplets from human to human through close contact. Populations at highest risk of developing pneumococcal infection include patients with high risk factors and health comorbidities. Patients who have any history of immunosuppression, chronic obstructive pulmonary disease, asplenia, and other chronic disease states are at high risk for developing pneumococcal infection.

Specifically, pneumococcal vaccinations provide preventative measures in protecting patients of all ages and those at high risk for acquiring the disease. The two pneumococcal vaccinations available in the United States are the pneumococcal conjugate vaccine (PCV13) and pneumococcal polysaccharide vaccine (PPSV23). Current recommendations for pneumococcal vaccinations provided by Center for Disease Control include the PPSV23 in high-risk adults from 18-64 years of age and for all adults 65 and older, and the PCV13 in certain high-risk populations in the United States (Centers for Disease Control and Prevention [CDC], 2017). Studies relating to PPSV23 and PCV13 efficacy demonstrate high estimates of clinical effectiveness and safety for both vaccines. Overall, the PPSV23 vaccine has shown approximately $70 \%$ clinical effectiveness and PCV13 has shown $75 \%$ clinical effectiveness in preventing invasive disease caused by the bacterial serotypes (CDC, 2017). 
The Use of DMAIC to Improve Quality Vaccination Recommendations in Chain Community Pharmacies

Even with such high levels of efficacy in these vaccines, pneumococcal vaccination rates in both the U.S. and the state of Tennessee are currently below the desired benchmarks to improve health outcomes. A number of factors contribute to such suboptimal vaccination rates with vaccine hesitancy being a top priority on the list. The World Health Organization lists vaccine hesitancy as one of the top 10 threats to global health outcomes in 2019 (Lo \& Hotez, 2017). Vaccine hesitancy has been defined as "a delay in vaccination or a refusal to vaccinate in spite of vaccine availability" (Reifler et al., 2016). The majority of vaccine hesitancy research has been conducted in the pediatric population; however, there has been a growing interest in hesitancy in the adult population, a field that is currently under researched. 
The Use of DMAIC to Improve Quality Vaccination Recommendations in Chain Community

Pharmacies

\section{Chapter 2: Review of the Literature}

The National Health Interview Survey (NHIS) is one of the major health surveys of the National Center for Health Statistics, which is a principal source for gathering information on the health of the noninstitutionalized civilian population of the United States (2020). NHIS collects data on a broad range of health topics by conducting personal interviews in households with adults throughout the U.S. to express a sample size representative of the national population. The methods and design for the NHIS are continuously reevaluated and refined to assure accurate data representation. The 2017 NHIS data show that pneumococcal vaccination coverage among adults aged 19-64 years at high risk for pneumococcal disease is $24.5 \%$ and $69.0 \%$ among adults aged 65 years and older in the U.S.

According to Healthy People 2020, the national objective is to increase the percentage of pneumococcal vaccinations in noninstitutionalized high-risk adults aged 19-64 years of age by $90 \%$ and in noninstitutionalized adults aged 65 and older by $60 \%$. In 2017, $74.1 \%$ of adults sixty-five or older received the PPSV23 vaccine in Tennessee. This number was above the national average of $72.4 \%$ for adults sixty-five or older (CDC, 2020). In 2017, 34.9\% of highrisk individuals less than sixty-five years old received the pneumococcal vaccine in Tennessee. This number was below the national average of $36.1 \%$ of high-risk individuals less than sixtyfive years old. As the current rates in both the U.S. and the state of Tennessee are currently below this metric, a number of factors contribute to such suboptimal vaccination rates. These factors include, but are not limited to, patient hesitancy, lack of strong provider recommendation, lack of access due to high costs, and lack of health awareness and information. The majority of these factors exist primarily in the outpatient setting. The high prevalence of such factors to exist has 
led to vaccines incompliance and is responsible for current, suboptimal pneumococcal vaccination rates. Researchers have tried to understand the basis of vaccination incompliance and found that to improve vaccine compliance, the following actions must be taken by health providers: provide patients educational material, use proven communication strategies, understand and clear misconceptions, improve accessibility for vaccination, make strong recommendations, and provide evidence that support the safety and effectiveness of vaccinations (Ventola, 2016).

Various studies have been conducted to determine the relationship between the strength of the provider's recommendation for vaccinations and patient intentions to receive a vaccine, and these studies found a strong association correlating strength of provider recommendation to patient vaccinations. Many other studies also show that strong recommendation technique is highly critical for the protection and prevention for all populations, especially the high risk for pneumococcal related diseases and outcomes. 


\section{Chapter 3: Methodology}

Research question

How can the use of Lean Six Sigma and the DMAIC process help improve pneumococcal vaccination rates in community pharmacy settings?

\section{Population}

The research includes gathering patient vaccination data from 54 community Walgreens pharmacies in the West Tennessee area. As part of this research, qualitative data through phone interviewing from community pharmacists in the selected Walgreen pharmacies were conducted.

\section{Study Methods}

Data collection used a multi-methods approach, including pharmacy dispensing data and indepth, semi-structured interviews with pharmacist key informants from the study sites. The design and implementation of the training program is geared towards Walgreens community pharmacists. Pneumococcal vaccination data were collected before and after the implementation of the training program followed by data analysis to determine the impact of the implementation. Qualitative data analysis included interviews that were conducted by trained student research assistants over the telephone, recorded, and subsequently transcribed verbatim by a third-party transcription service to evaluate the usefulness of the skills learned from the training. Quantitative data analysis included both descriptive and inferential statistics, including chisquare to test the primary objective. This research will utilize a quality improvement process and lean six sigma methodologies to assist with the primary objective. 
The Use of DMAIC to Improve Quality Vaccination Recommendations in Chain Community

Pharmacies

\section{Chapter 4: LSS and The DMAIC Methodology}

LSS and DMAIC (Define, Measure, Analyze, Improve, Control) methods have been used in this research to focus on improving specific existing challenges in the vaccine

recommendation process. LSS methods have been more commonly integrated within healthcare in the past decade. In an early 2020 study, LSS has been used to improve access to care in surgical subspecialty clinics (Flanary et al., 2020). In an area where a delay to new patient visits and consults challenged the care for patients, LSS was implemented to improve the timeliness of care for patients and reduce its variation by improving clinic processes and scheduling.

LSS has been a model used in other aspects of healthcare as well such as pharmacy. While pharmacy is a demanding, patient- driven field, many of its issues lie within patient experiences and inefficiencies that ultimately lead to patient dissatisfaction. Recently in a 2018

study, LSS was incorporated into pharmacy practice to reduce pharmacy wait time. LSS methods in this study were used to identify potential problems associated with increased wait times and found that implementations such as automated queuing technology, pharmacy devices for quick and accurate filling and dispensing, and computer simulation modeling for smooth workflow were solutions to the issue (Alam, 2018). LSS methodologies have been utilized in several settings to reduce patient wait time, attract new customers, decrease workload and improve the overall efficiency within the organization.

DMAIC is an aspect within LSS that includes defining a problem, quantifying the problem, analyzing the cause of the problem, implementing a feasible solution, and maintaining the solution. The integration of DMAIC in this research provides a well-guided approach to help 
The Use of DMAIC to Improve Quality Vaccination Recommendations in Chain Community

Pharmacies

improve the efficiency and performance within the process of vaccination recommendation and assertiveness. The implementation of the DMAIC process has been utilized to address the standards and approach currently used in the pneumococcal vaccination recommendation process at participating Walgreens pharmacies. 
The Use of DMAIC to Improve Quality Vaccination Recommendations in Chain Community Pharmacies

\section{Chapter 5: DMAIC Case Study}

Define

The problem of interest is pneumococcal vaccine rates below specification in community pharmacies. Specifications were based in Healthy People 2020, rather than traditional voice of consumer (VoC) analyses given the uniqueness of the product of interest (i.e. a vaccine). A high-level map of vaccine recommendation processes, as shown in Figure 1, was conducted to determine the key factors affecting vaccine recommendation. With Walgreens divisional leadership, the objective to increase pneumococcal vaccination rates across Tennessee by $20 \%$ year-over year was set and tested in study site pharmacies.

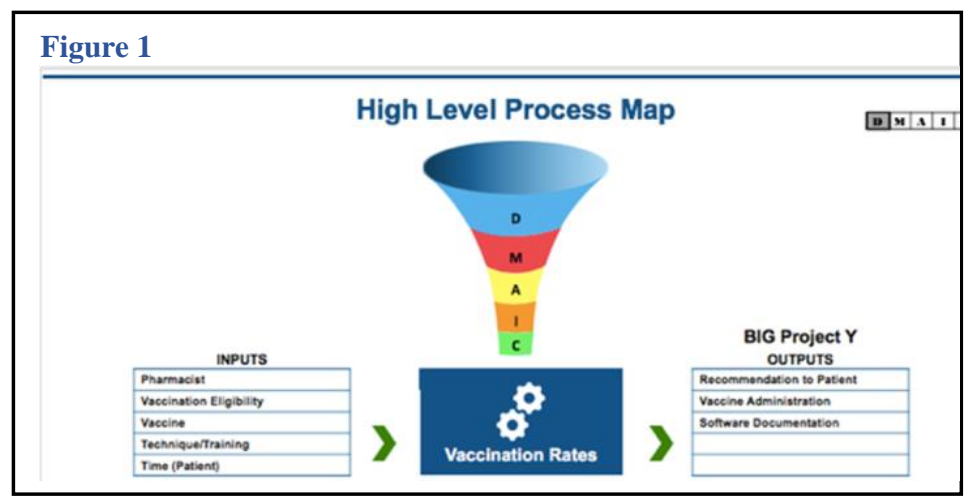

Measure

Base line (e.g. "current state") data from the dispensing history for pneumococcal vaccinations administered was gathered from each pharmacy and was compared across the pharmacy division. Key informant interviews were conducted to provide context on processes in community pharmacy workflow. 
The Use of DMAIC to Improve Quality Vaccination Recommendations in Chain Community Pharmacies

Analyze

Baseline data from historical pharmacy dispensing data was used to understand correlations between staffing and hours of operations and vaccination rates, but no significant correlations were identified after conducting a regression analysis. Content analysis of interview transcriptions highlighted vaccination hesitancy and related barriers present during pharmacist recommendations. A cause-and-effect matrix along with a failure modes and effects analysis (FMEA) were conducted to determine the potential root cause and determinant to the overarching issue and are shown in Figures 2 and 3, respectively. As a result, the vaccine recommendation communication technique used by the pharmacist was selected as the root cause hypothesis.

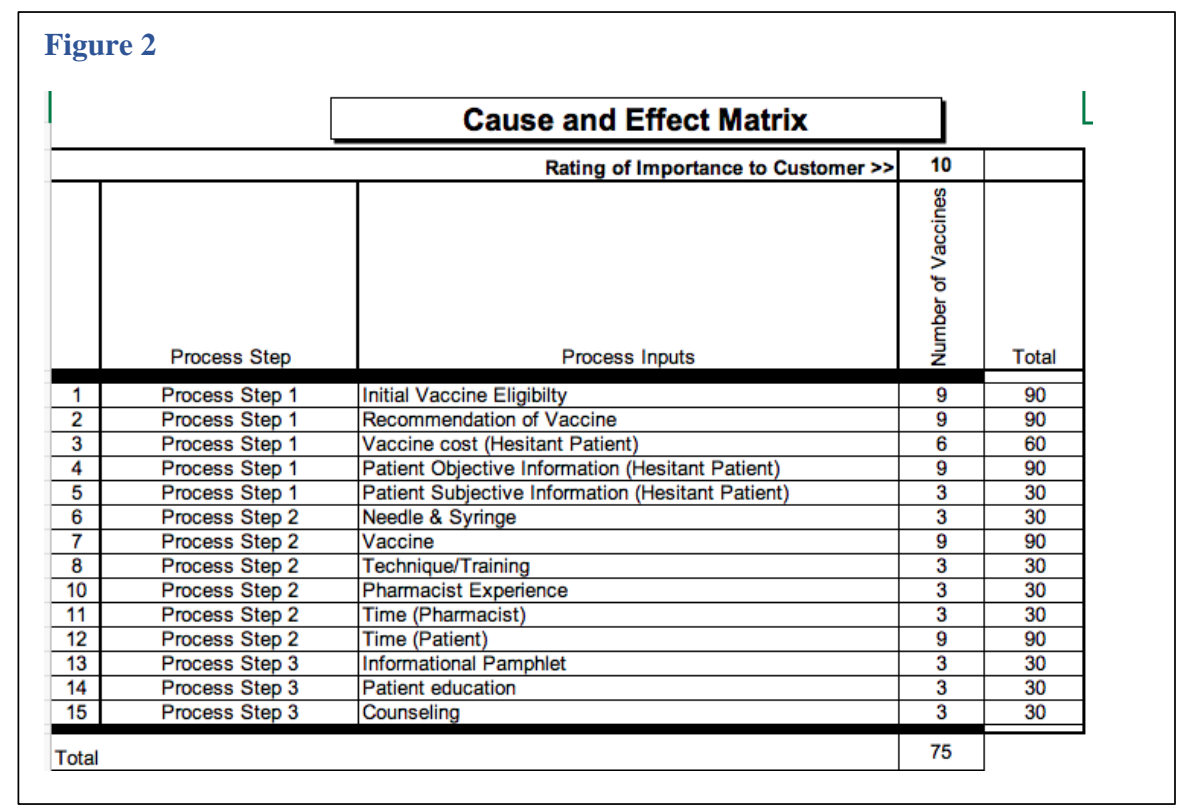




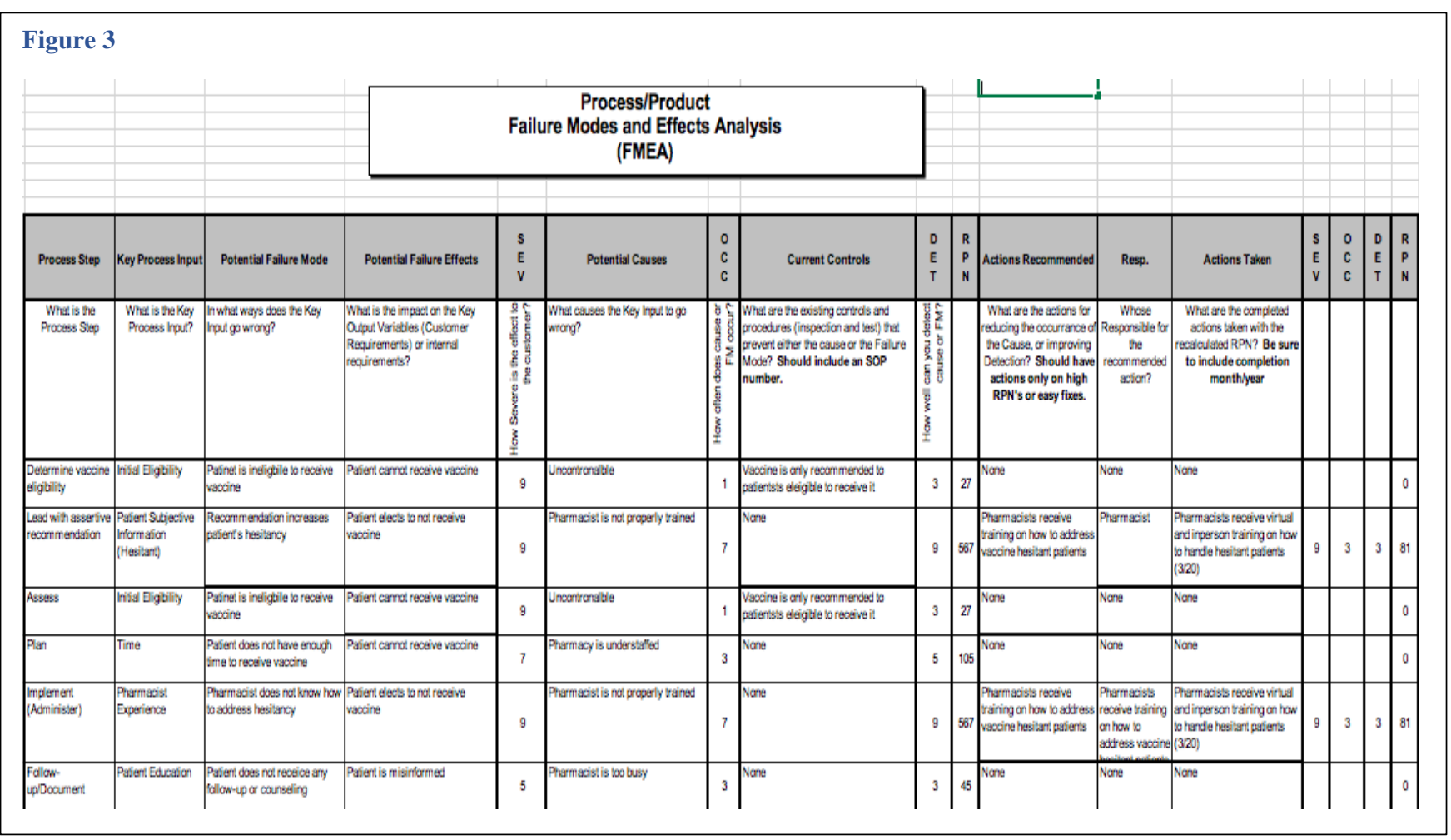

\section{Implement}

To improve the recommendation communication technique, implementing a training program has been decided to potentially be the most feasible and sound way to educate and improve vaccine rates in pharmacies. The intent of the training program is to focus on educating and training pharmacists on vaccine assertiveness technique and will be implemented within the community pharmacies study sites. The training was directed towards study site pharmacists representing and consisted of both online and live training sessions. The training program centered on the concept of "presumptive" vaccine recommendations motivational interviewing and the transtheoretical model to guide pharmacists through behavior change toward vaccine recommendation acceptance. The live sessions were led by faculty facilitators and included 
The Use of DMAIC to Improve Quality Vaccination Recommendations in Chain Community Pharmacies

practice of key verbiage and mock sessions to practice improving the communication of vaccine recommendations.

\section{Results of the Implementation}

The results of the study were gathered through a variety of statistical tests to test the effectiveness of the training program. The Chi Square test, represented in Figure 4, was used for unpaired, bivariate data which represents the dataset of pneumococcal vaccines preimplementation and post implementation. Thereafter, a capability analysis test was conducted to evaluate the process capability, $\mathrm{Cp}, \mathrm{Cpk}$, and p-value. A total of 54 pharmacies completed the experiment, including 25 intervention sites. Of these, 8 increased by $20 \%$. Of the 29 control sites that did not receive training, 4 increased by the specification of $20 \%$. Year-over-year comparison of mean pneumococcal vaccination rates showed no significant difference $(\mathrm{p}<0.05)$. The new processes capability analysis was run as a one-sided analysis with a goal and LSL of $20 \%$ to compare the 2018 vs 2019 vaccination rates, as shown in Figure 5. The analysis revealed $70.49 \%$ of the stores fell below the LSL and did not achieve the goal of increasing vaccinations by $20 \%$, as displayed in the process capability report in Figure 6. To show the results in a simpler format, refer to Graph A below. 
The Use of DMAIC to Improve Quality Vaccination Recommendations in Chain Community

Pharmacies

Graph A

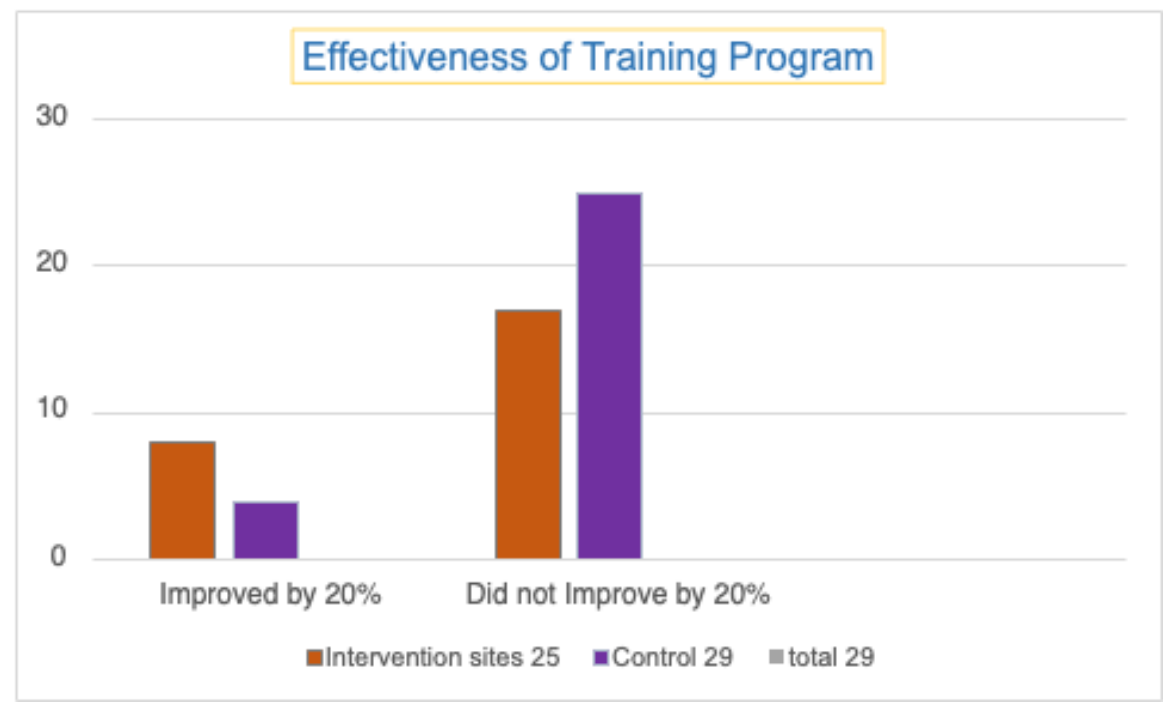

Figure 4

\begin{tabular}{|c|c|c|}
\hline \multicolumn{3}{|c|}{ Chi-Square Two-Way Table Statistics } \\
\hline Observed Counts & Achieved Goal & Below Goal \\
\hline Intervention & 8 & 17 \\
\hline Control & 4 & 25 \\
\hline Expected Counts & Achieved Goal & Below Goal \\
\hline Intervention & 5.556 & 19.444 \\
\hline Control & 6.444 & 22.556 \\
\hline Std. Residuals & Achieved Goal & Below Goal \\
\hline Intervention & 1.037089946 & -0.554348 \\
\hline Control & -0.962914 & 0.514699 \\
\hline Chi-Square & 2.575 & \\
\hline DF & 1 & \\
\hline P-Value & 0.1086 & \\
\hline
\end{tabular}


The Use of DMAIC to Improve Quality Vaccination Recommendations in Chain Community

Pharmacies

Figure 5
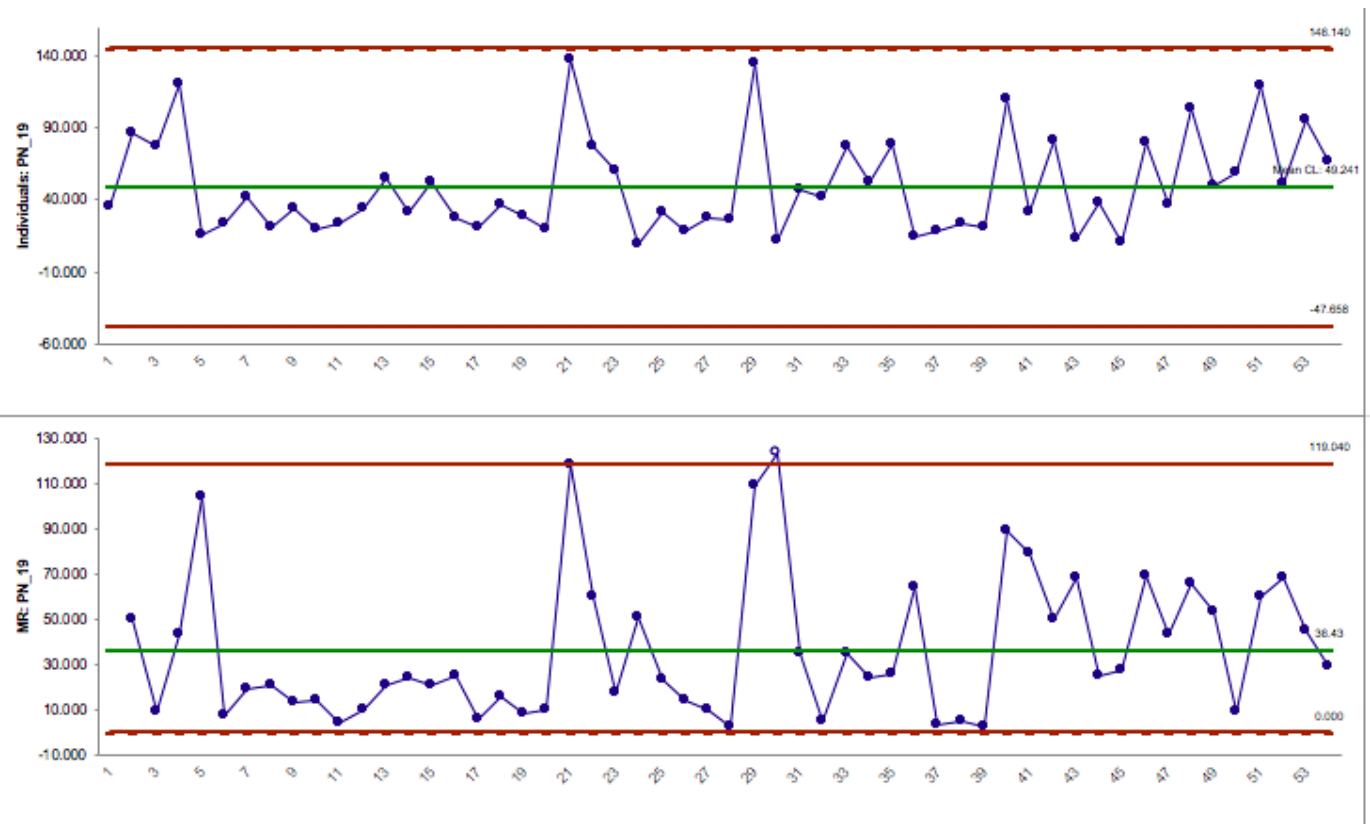

Figure 6

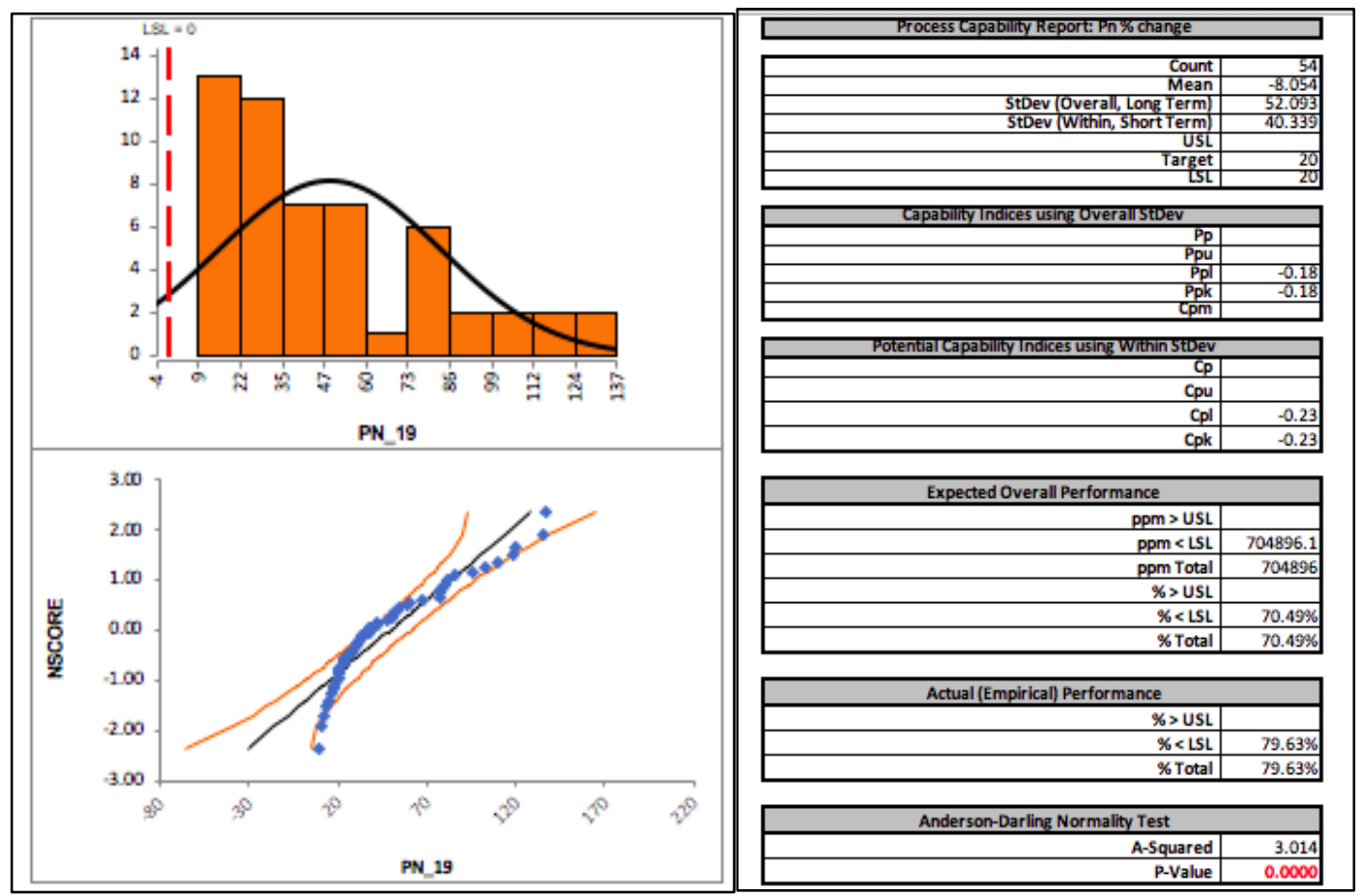


The Use of DMAIC to Improve Quality Vaccination Recommendations in Chain Community

Pharmacies

\section{Control}

After conducting the training program with pharmacists, trends in pneumococcal vaccination rates over the course of a year at each pharmacy will be monitored. This will help evaluate the skills and assertive techniques that were adapted and integrated by each pharmacist at each Walgreens pharmacy. At the end of the training program, pharmacists will be provided "Reference Guides" of the vaccine recommendation process, as represented in Figure 7, to attach to their computer monitors at their respective sites to remind and ensure each eligible vaccine recommendation is strong and assertive. Adherence to these guides will be a fidelity outcome and will help to evaluate the extent to which each pharmacy adapted to the lessons learned from the training. Finally, follow up interviews will be performed with pharmacists postimplementation to discuss the strengths and weaknesses of the training and evaluate the usefulness of the skills learned from the training sessions.

Figure 7

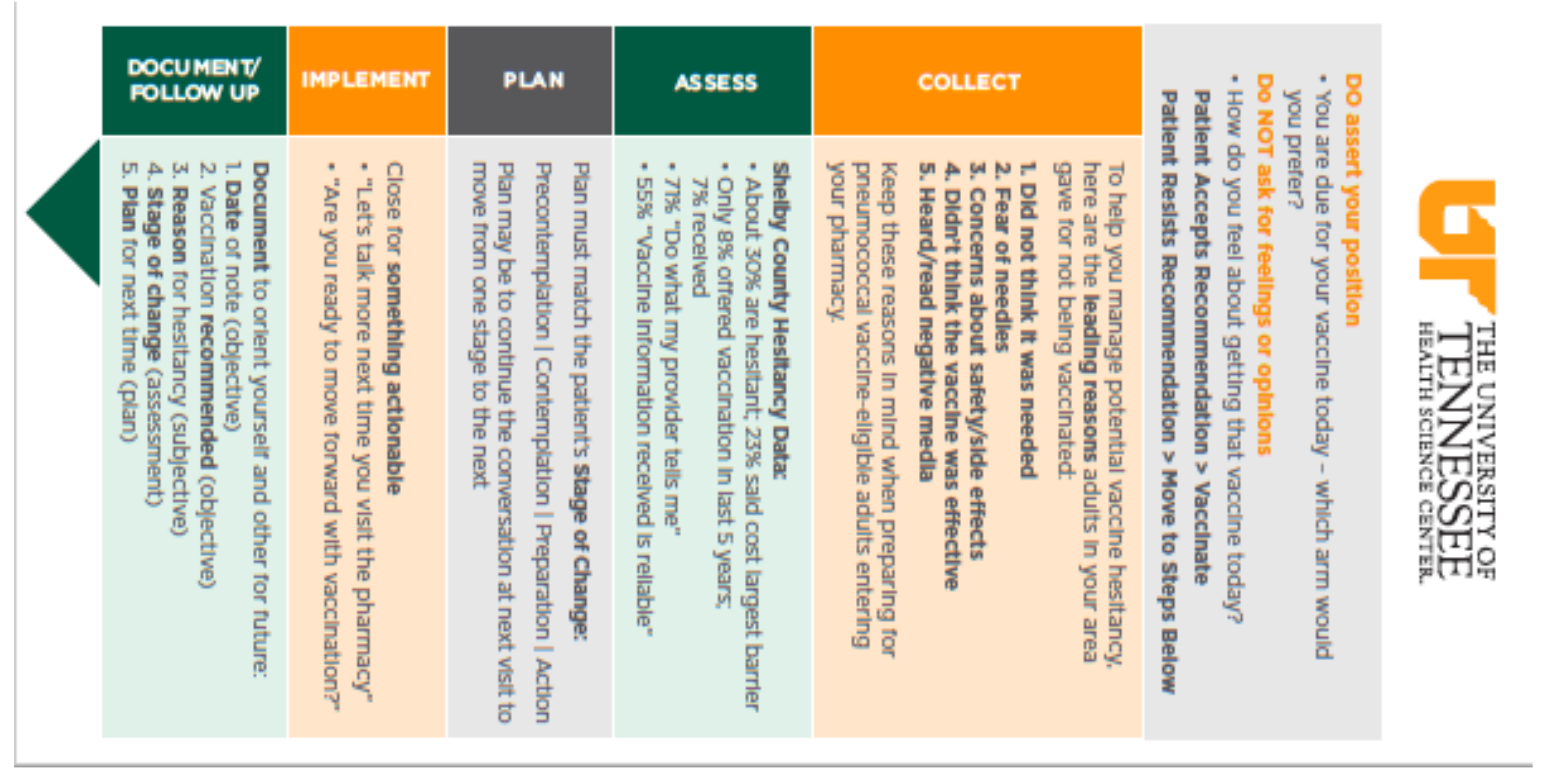


The Use of DMAIC to Improve Quality Vaccination Recommendations in Chain Community

Pharmacies

\section{Chapter 6: Discussion}

The primary focus of training program was to improve the quality of pneumococcal vaccine recommendations pharmacists provide in the community setting to increase the overall rates of vaccinations and optimize health outcomes. Research has suggested that the influence of quality recommendations has a significant impact on vaccine behaviors and vaccine initiation. In a prior research study examining the correlation between the quality of a vaccine recommendation to vaccine initiation, researchers found that receiving a high-quality recommendation versus no recommendation was associated with over nine times the odds of having received the vaccination and four times the odd compared to a low-quality recommendation (Gilkey et al., 2016). The study also found that higher recommendation quality was associated with less vaccination refusal and delay. It is quite evident that higher quality recommendations significantly influenced the decision making of receiving the vaccination and led to higher overall initiation rates.

\section{Limitations and Insights}

While the outcomes of the training program did not result as expected a discussion to help identify various factors that may have contributed to the variable outcomes of the training program can help understand and address the shortcomings of this research. To understand both the factors of success and the potential barriers to implementing and sustaining the implementation of the training program, we used the Consolidated Framework for Implementation Research (CFIR) to help evaluate various factors in a systematic approach. We examined each domain of the CFIR construct such as intervention characteristics, outer setting, 
The Use of DMAIC to Improve Quality Vaccination Recommendations in Chain Community

inner setting, characteristics of individuals, and process of implementation to consider issues that both facilitated and hindered implementation.

When considering intervention characteristics, several relative advantages of the implementation were noted. The training program intended to equip pharmacists with stronger skills in their communication and assertive techniques to improve pneumococcal vaccine rates and improve overall patient health. The training program was adaptable to all community pharmacies where there is convenience in providing vaccinations and feasibility to implement in routine practice.

Regarding outer setting, patient resources were considered in terms of if there was a willingness for patients to engage in active discussion during a vaccine recommendation. External policies such as insurance coverage can influence patients' decision to receive vaccinations. Another important consideration is the pre-administration processing time of a vaccination which can influence and defer patients from receiving vaccinations at the pharmacy

In terms of inner setting, understanding the culture, implementation climate, and readiness for implementation were critical. Community pharmacies, in general, are sometimes perceived as environments of high tension where pharmacists are challenged with multiple initiatives and benchmarks that are considered more important than others. And as a common result, more efforts are typically directed to initiatives that are incentivized versus those that are not. Time and workload were other barriers commonly seen that may have impacted the climate of learning new vaccine recommendation techniques and integrating within practice. 
In terms of characteristics of individuals, it is valuable to consider the knowledge and beliefs about the intervention, and vaccinations in general. The intended purpose of the training program was to improve pharmacist recommendation technique, expand patient education, and help dissolve several hesitancy factors including culture and misconceptions. While acquiring new knowledge and further solidifying important strategies, attitudes and perception play an important role in a new implementation. That is why we may consider another barrier to successful implementation: the resistance to change in routine and resolute perceptions.

Lastly, we may reassess the process of implementation: the planning and execution of the training program. Perhaps another reason for unsuccessful improvement was simply a weak implementation of the program or weak integration of techniques within the pharmacies after implementation. Perhaps, our strategies may have not been strong enough to adapt firmly and integrate within the participating pharmacies. As a means of reflecting and evaluating the effectiveness of the training program, we conducted interviews with pharmacists that participated in the training post implementation. From the interviews, we acquired qualitative data and gained perspective on aspects of the program that were integrated successfully as well as measures for quality improvement. Future studies may focus on the strategy of implementation and improvement to better correlate an assertive training program for pharmacists to improve vaccination rates in the community setting.

\section{Conclusion}

Though quantitative data did not suggest a strong enough correlation to determine the impact of the training program to the overall increase in pneumococcal vaccination rates, this 
research provides future direction on how community pharmacists can better facilitate vaccine recommendations and be key players in improving overall vaccination rates to better patient outcomes. In regard to the data analysis, we found settings where the training program was beneficial and others where they were not. Considering several influential factors that may be outside of the study's control, it is difficult to determine whether or not the training program positively or negatively impacted pneumococcal vaccination rates in community pharmacies. Perhaps, the lessons learned from this research study can provide future researchers and pharmacy practitioners a direction for the future to overcome vaccine hesitancy and improve quality recommendations. 
The Use of DMAIC to Improve Quality Vaccination Recommendations in Chain Community

Pharmacies

\section{References}

1. Alam, S., Osama, M., Iqbal, F., \& Sawar, I. (2018). Reducing pharmacy patient waiting time. International journal of health care quality assurance. https://doi.org/10.1108/IJHCQA-08-2017-0144

2. Almeida, A. F., Sobrinho-Simões, J., Ferraz, C., Nunes, T., \& Vaz, L. (2016). Pneumococcal pneumonia vaccine breakthroughs and failures after 13-valent pneumococcal conjugated vaccine. The European Journal of Public Health, 26(5), 887889. https://doi.org/10.1093/eurpub/ckw089

3. American Journal of Managed Care (2018). Addressing barriers to optimal communitybased vaccination. Retrieved from www.ajmc.com/journals/supplement/2018/bolsteringvaccine-use/addressing-barriers-to-optimal-communitybased-vaccination.

4. Centers for Disease Control and Prevention. (2017). About pneumococcal vaccine. https://www.cdc.gov/vaccines/vpd/pneumo/hcp/about-vaccine.html.

5. Centers for Disease Control and Prevention. (2020). National Center for Immunization and Respiratory Diseases. https://www.cdc.gov/ncird/index.html.

6. Centers for Disease Control and Prevention. (2020). National health interview survey. https://www.cdc.gov/nchs/nhis/index.htm.

7. Dempsey, A. F., Pyrzanowski, J., Lockhart, S., Campagna, E., Barnard, J., \& O'Leary, S. T. (2016). Parents' perceptions of provider communication regarding adolescent vaccines. Human vaccines \& immunotherapeutics, 12(6), 1469-1475. https://doi.org/10.1080/21645515.2016.1147636

8. Flanary, J. T., Rocco, N. R., Dougherty, T., \& Christman, M. S. (2020). Use of Lean Six Sigma to Improve Access to Care in a Surgical Subspecialty Clinic. Military

Medicine, 185(5-6), e887-e893. https://doi.org/10.1093/milmed/usz426

9. Gilkey, M. B., Calo, W. A., Moss, J. L., Shah, P. D., Marciniak, M. W., \& Brewer, N. T. (2016). Provider communication and HPV vaccination: The impact of recommendation quality. Vaccine, 34(9), 1187-1192. https://doi.org/10.1016/j.vaccine.2016.01.023

10. Golos, M., Eliakim-Raz, N., Stern, A., Leibovici, L., \& Paul, M. (2019). Conjugated pneumococcal vaccine versus polysaccharide pneumococcal vaccine for prevention of pneumonia and invasive pneumococcal disease in immunocompetent and immunocompromised adults and children. The Cochrane Database of Systematic Reviews, 2019(2), CD012306. https://doi.org/10.1002/14651858.CD012306.pub2

11. Grabenstein, J. (1998). Pharmacists as vaccine advocates: Roles in community pharmacies, nursing homes, and hospitals. Vaccine.16(18), 1705-10. https://doi.org/10.1016/S0264-410X(98)00131-5

12. Healthy People 2020. (2020). Immunization and infectious diseases. https://www.healthypeople.gov/2020/topics-objectives/topic/immunization-andinfectious-diseases/objectives.

13. Henrikson, N. B., Opel, D. J., Grothaus, L., Nelson, J., Scrol, A., Dunn, J., Faubion, T., Roberts, M., Marcuse, E. K., \& Grossman, D. C. (2015). Physician Communication Training and Parental Vaccine Hesitancy: A Randomized Trial. Pediatrics, 136(1), 7079. https://doi.org/10.1542/peds.2014-3199 
The Use of DMAIC to Improve Quality Vaccination Recommendations in Chain Community

Pharmacies

14. Lo, N. C., \& Hotez, P. J. (2017). Public health and economic consequences of vaccine hesitancy for measles in the United States. JAMA pediatrics, 171(9), 887-892. doi:10.1001/jamapediatrics.2017.1695

15. MacDonald, N. E., Butler, R., \& Dubé, E. (2018). Addressing barriers to vaccine acceptance: an overview. Human vaccines \& immunotherapeutics, 14(1), 218-224. https://doi.org/10.1080/21645515.2017.1394533

16. Omer, S. B., Salmon, D. A., Orenstein, W. A., Dehart, M. P., \& Halsey, N. (2009). Vaccine refusal, mandatory immunization, and the risks of vaccine-preventable diseases. New England Journal of Medicine, 360(19), 1981-1988. https://www.nejm.org/doi/full/10.1056/nejmsa0806477

17. Opel, D. J., Heritage, J., Taylor, J. A., Mangione-Smith, R., Salas, H. S., Devere, V., Zhou, C., \& Robinson, J. D. (2013). The architecture of provider-parent vaccine discussions at health supervision visits. Pediatrics, 132(6), 1037-1046. https://doi.org/10.1542/peds.2013-2037

18. Powers, M. F., \& Bright, D. R. (2008). Pharmacy technicians and medication therapy management. Journal of Pharmacy Technology, 24(6), 336-339. https://doi.org/10.1177/875512250802400604

19. Reifler, J., Richey, S., \& Freed, G. L. (2016). Time to vaccinate against hesitancy. Vaccine, 34, 6700-6706. https://doi.org/10.1038/s41591-019-0524-1

20. Taitel, M., Cohen, E., Duncan, I., \& Pegus, C. (2011). Pharmacists as providers: targeting pneumococcal vaccinations to high-risk populations. Vaccine, 29(45), 8073-8076. https://doi.org/10.1016/j.vaccine.2011.08.051

21. Ventola C. L. (2016). Immunization in the United States: Recommendations, Barriers, and Measures to Improve Compliance: Part 1: Childhood Vaccinations. $P \&$ T: a peerreviewed journal for formulary management, 41(7), 426-436.

22. Vyas, D., Galal, S. M., Rogan, E. L., \& Boyce, E. G. (2018). Training Students to Address Vaccine Hesitancy and/or Refusal. American journal of pharmaceutical education, 82(8). https://www.ncbi.nlm.nih.gov/pmc/articles/PMC4927017/

23. World Health Organization. Pneumococcal vaccines WHO position paper--2012. Weekly Epidemiological Record 2012; Vol. 87:129-44. 